\title{
COVID-19 vaccine-induced acute generalized exanthematous pustulosis
}

\author{
Sung-Yoon Kang ${ }^{1}$, So-Young Park ${ }^{2}$, Jung-Hyun Kim${ }^{3}$, Sang Min Lee ${ }^{1}$, and Sang Pyo Lee
}

\author{
${ }^{1}$ Department of Internal Medicine, \\ Gachon University Gil Medical \\ Center, Incheon; ${ }^{2}$ Division of \\ Pulmonary, Allergy and Critical Care \\ Medicine, Department of Internal \\ Medicine, Konkuk University \\ Medical Center, Seoul; ${ }^{3}$ Department \\ of Internal Medicine, Armed Forces \\ Capital Hospital, Seongnam, Korea
}

Received: April 21, 2021

Revised : May 4, 2021

Accepted: May 16, 2021

\section{Correspondence to}

Sung-Yoon Kang, M.D.

Tel: +82-32-460-3201

Fax: +82-32-469-4320

E-mail: allergy4u@gmail.com https://orcid.org/0000-00015505-3028
A previously healthy 32-year-old woman presented to the outpatient allergy clinic with a generalized pruritic rash and feverish sensation 3 weeks after receiving her first dose of a coronavirus disease 2019 (COVID-19) vaccine (ChAdOx1 nCoV-19 vaccine, AZD1222).

She had pustular eruptions atop erythematous areas that started in the axillary regions and rapidly progressed to the upper and lower extremities. Upon examination of her upper extremities, we noted various sizes of erythematous lesions containing numerous pinhead-sized pustules (Fig. 1A). She had no history of concomitant medications, viral infections, or drug allergies either before or after the COVID-19 vaccination.

Laboratory findings revealed a white blood cell count of $12.72 \times 10^{3} / \mathrm{mm}^{3}$ with
$89.0 \%$ neutrophils. She was initially treated with an oral antihistamine and high-potency topical corticosteroids. For symptomatic relief of pruritus and skin inflammation, we added a short course of systemic corticosteroids. The disseminated nonfollicular pustules disappeared with desquamation within 4 days (Fig. $1 B$ ), and the skin lesions resolved within 2 weeks.

Acute generalized exanthematous pustulosis (AGEP) is a rare cutaneous reaction characterized by the acute onset of nonfollicular pustules on an erythematous base. AGEP is frequently associated with drugs, primarily antibiotics, followed by viral infections, ultraviolet radiation, and heavy metals. Per the patient's request, we did not perform a skin biopsy to rule out other pustular eruptions or patch
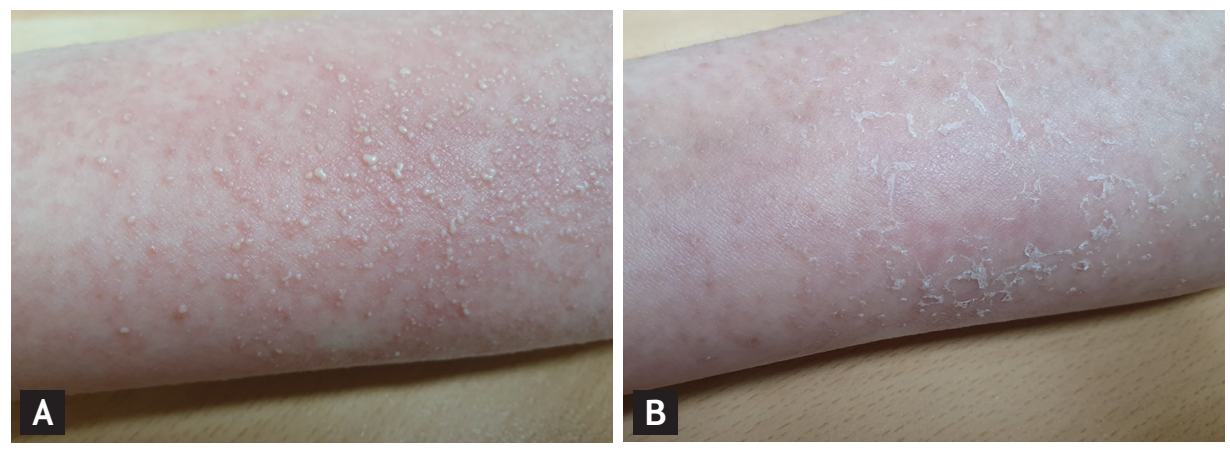

Figure 1. Clinical features of acute generalized exanthematous pustulosis (AGEP) after coronavirus disease 2019 (COVID-19) vaccination. Multiple nonfollicular pustules on an erythematous background were observed on the upper extremities (A). Four days following treatment with topical and systemic corticosteroids and an oral antihistamine, most of the pustules had rapidly resolved with desquamation (B). 
tests to search for the causative agent. Nevertheless, her cutaneous manifestations and clinical course were consistent with AGEP; based on her AGEP validation score, we made a probable diagnosis of AGEP. To the authors' knowledge, this is the first report of COVID-19 vaccine-induced AGEP. There have been a few reports of AGEP following diphtheria-tetanus-pertussis, measles-mumps-rubella, influenza, and pneumococcal vaccinations, but its mechanism remains unknown. This study was approved by the Institutional Review Board of the Gachon University Gil Medical Center (IRB: GFIRB 2021-110). The need for informed consent was waived by the Institutional Review Board.

\section{Conflict of interest}

No potential conflict of interest relevant to this article was reported. 